\title{
Error patterns in judgment and production of horizontal and vertical scale positions
}

\author{
J. B. BROOKE and A. W. MacRAE \\ University of Birmingham, Birmingham B15 2TT, England
}

\begin{abstract}
Cyclic error curves are often found when people judge or set the position of a marker on a linear scale, but various different forms of cyclic pattern have been reported. Twelve subjects each made judgments and also made settings, both on a horizontal linear scale and on a vertical one. Analysis of their error patterns showed a strong tendency to generate the opposite pattern when the response mode changed from judgment to production of scale positions. This is interpreted as evidence that the errors arise from perceptual misjudgments rather than from response biases. Error patterns tended to remain constant for changes in scale orientation alone. Thus, the varying scale orientations do not seem to account for the reported discrepancies in pattern. However, it was found that different subjects could have opposite patterns in the same experimental condition. These were real individual differences because subjects' patterns tended to persist across changes in orientation and to reverse with changes in response mode. It seems likely that the reported differences in pooled error trends reflect different assortments of subjects who differ fundamentally in their perception of linear scale positions.
\end{abstract}

When people attempt to judge the position of a marker on a spatial interval, they make errors that are typically overestimates when the marker is in one half of the interval and underestimates when it is in the other. We adopt the convention of identifying as "lower" the half of the scale that is labeled with numerically smaller values, irrespective of the physical nature or orientation of the stimulus display. The direction of labeling is arbitrary, since errors of numerical overestimation occurring in the numerically lower half of the scale, along with underestimation in the upper half, always reflect a tendency for responses to be nearer the center of the range than they should be.

In order to characterize the different error curves found in experiments of this type, we use the term "positive cubic" to denote results where the subject's errors are towards the center of the range of responses and "negative cubic" when they are away from the center. The terms reflect our use of curve-fitting as a descriptive technique. Our descriptive analysis is explained in the Results section, and illustrated in Figure 1.

It is necessary to distinguish carefully between two kinds of experimental procedure. In judgment, an interval containing a marker is displayed and the

This work was supported by a research training award to J. B. Brooke by the Science Research Council (U.K.). Requests for reprints should be sent to Dr. J. B. Brooke, Department of Applied Psychology, UWIST, Penylan, Cardiff CF3 7UX, Wales, or to Dr. A. W. MacRae, Department of Psychology, University of Birmingham, P.O. Box 363, Birmingham B15 2TT, England. subject has to identify the marker's position, usually numerically. In production, the subject is given a numerical value and has to represent it by his setting of the marker within the interval. The importance of the distinction lies in its potential for distinguishing between perceptual and response biases as a source of error. Suppose that there is no response bias but there is a perceptual error such that the subject judges a stimulus to be $\mathrm{X}+\mathrm{i}$ where its true value is $X$. He thus makes a response which is too large and generates a positive error $\mathrm{i}$. When asked to produce the stimulus value $X+i$, he sets the marker to $X$ and thus generates a negative error $-i$. Thus, error patterns with the production procedure will be complementary to those found with judgment if the source of error is perceptual. If, on the other hand, errors arise from a tendency, say, to avoid extreme responses, then the same pattern of errors will be found with both procedures.

Although most experiments on interpolation in linear intervals have revealed either positive or negative cubic trends, there are rather marked inconsistencies between the results reported. Miller (1950) obtained judgments of the scale position represented by a marker within a horizontal interval. His subjects made errors which were predominantly of the negative cubic type. He presented a large number of stimulus items simultaneously on a card, and subjects could work through them at their own pace and with crosscomparisons if they wished. Levett (1952) repeated Miller's procedure and also presented analogous horizontal intervals individually, using a modified slide rule. He also used the additional procedure of getting subjects to produce pointer settings to 
represent scale values requested by the experimenter. Both forms of the judgment procedure gave negative cubic error patterns with pooled data, while the production procedure gave positive cubic curves. This result argues a perceptual source for the error, as noted earlier.

Nash (1964) used only a production procedure in which the interval was represented on a strip of paper placed horizontally but in a saggital plane, that is, appearing as a vertical column rather than as a transverse line to the subject looking down on it. In his experiment, the average result was a negative cubic curve, whereas Levett had found positive cubic error curves with his production procedure.

The discrepancy may perhaps be attributable to the different intervals that were used. Carr and Garner (1952) found a positive cubic error curve for judgments of a $25-\mathrm{mm}$ interval and a negative cubic one with a $.5-\mathrm{mm}$ interval. Although Miller had used intervals from 1 to $10 \mathrm{~mm}$ in total length, his data summaries do not permit separate examination of the different sizes. Levett used only a $10-\mathrm{mm}$ interval in all three procedures, whereas Nash used an interval of 8.33 in. ( $21 \mathrm{~mm})$. Thus, it is possible that Nash's discrepant result was attributable to the greater length of his interval. However, his procedure differed also in using a vertical rather than a horizontal interval, and this may be its critical feature.

In experiments on the judgment of interpolations along the radii of concentric circles on simulated radar displays, Chapanis (1947) and Chapanis and Leyzorek (1950) obtained positive cubic error curves. Bartlett, Reed, and Duvoisin (1949) and Leyzorek (1949) found very little discernible pattern in the errors.

These experiments used a variety of orientations and of interpolation intervals but, unfortunately for our purposes, the shapes of the error curves seem not to have been obtained for individual stimulus conditions of individual subjects. Miller (1950) considered that individual differences in performance were of greater influence than interval size. Brooke and MacRae (1977) found that, with judgments of numerical proportion (a judgment dimension having bounded properties analogous to a linear interval), consistent individual differences existed in error pattern.

The present study thus addressed the following questions: (1) Do errors of interpolation show a systematic relation to position in the interval? (2) Do these errors depend on the orientation of the interval? (3) Do the errors arise from perceptual or from response processes? (4) Do individuals show characteristic patterns of error?

\section{METHOD}

Subjects either judged a marker's position on a linear interval or adjusted a marker on a linear interval to represent scale values.
The interval was oriented either vertically or horizontally. There were, thus, four experimental conditions: vertical judgment, vertical production, horizontal judgment, and horizontal production.

Stimuli were generated on a Tektronix 611 visual display unit by a PDP-11 computer. In the conditions with the horizontal scale, the display showed two vertical lines, $3 \mathrm{~mm}$ long, separated by a horizontal distance of $13 \mathrm{~mm}$. These represented positions 0 and 21 on a horizontal scale. Scale values were indicated by a dot, which could take up any position along an imaginary horizontal line extending between the markers. A number from 00 to 21 , in digits $9 \mathrm{~mm}$ high, was displayed about $15 \mathrm{~mm}$ above and to the right of the interval. The display was the same in the conditions with the vertical interval except that the scale was one of vertical position and was bounded in positions 0 and 21 by $3-\mathrm{mm}$ horizontal lines.

In the two judgment conditions the dot was displayed by the computer in a position representing one of the scale values, 1-20, and the subject was required to judge it. He made his response by adjusting a 10-turn potentiometer. This controlled a voltage which was read by the computer and was used to adjust the displayed number to any integer from 01 to 20 . When the subject was satisfied that the displayed number corresponded to the position of the dot on the scale, he pressed a button. This caused his setting to be recorded, and the computer displayed the next stimulus.

In the two production conditions, the computer selected and displayed a number. The subject's task was to adjust the dot to the corresponding scale position by means of the potentiometer. When the subject pressed the response button, his setting of the dot position was recorded.

\section{Subjects and Design}

Five female and seven male undergraduate student volunteers were each paid $£ 1$ for participating. Each encountered all four of the experimental conditions, but in different orders.

There are 24 possible orders in which four conditions can be presented. Twelve of these were chosen such that each condition occurred equally often in each ordinal position and each condition followed each other condition equally often. Each subject was randomly assigned to a different one of these permutations.

Each subject served for two sessions of about $\mathbf{4 0} \mathrm{min}$ each and separated by at least a day. Each session consisted of two conditions of the experiment, each preceded by a demonstation of the positions of the various points on the scale. Each condition of the experiment involved $\mathbf{8 0}$ trials, and the subject completed a condition without any scheduled rest periods, though the responses were self-paced; there was a short rest period between the two conditions of any session.

Stimuli were chosen according to a prearranged sequence in which each occurred equally often. This sequence was entered at different points in the four conditions any subject performed, and the 12 subjects in a condition entered it at eight different points.

\section{RESULTS}

As noted earlier, several authors have reported systematic patterns of constant error in interpolation experiments. However, they have virtually always chosen to pool data across subjects in order to show these trends clearly. In our view, such pooling is undesirable and misleading. We require a quantitative index for each subject of any tendency for responses to be too extreme or too close to the midpoint.

Our solution is to find the value of the parameter c which gives the best fit to the data of the cubic curve: 

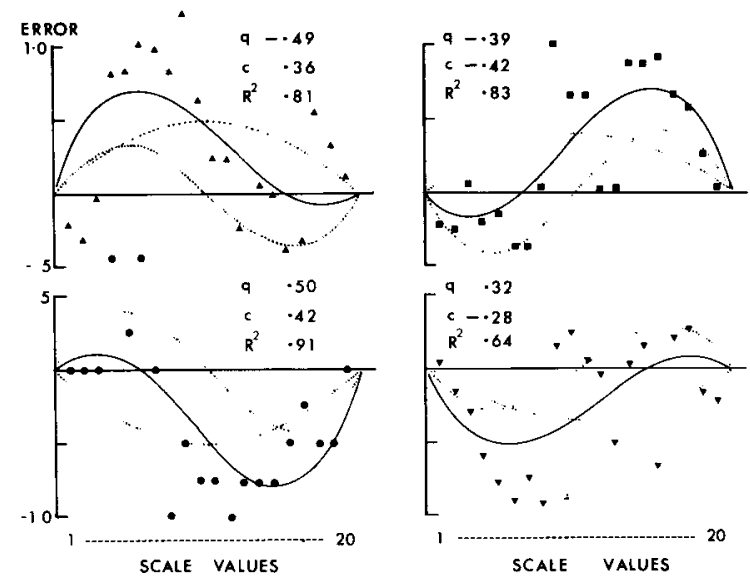

Figure 1. Constrained quadratic and cubic curves (dotted lines) fitted to four typical sets of data. Each point represents the mean of four errors made by one subject on one experimental condition and at one position on the interval. The signs and magnitudes of the coefficients $q$ and $c$ together specify the characteristic shape of the best-fitting combined function (continuous line). The usual multiple regression coefficient of determination $R^{2}$ represents the proportion of variance in the plotted points accounted for by the combined curve.

$$
\hat{\mathrm{Y}}=\mathrm{cA}(\mathrm{X}-\mathrm{L})(\mathrm{X}-\mathrm{M})(\mathrm{X}-\mathrm{H}) \text {. }
$$

$\mathrm{X}$ and $\hat{\mathrm{Y}}$ represent scale value and corresponding predicted error, respectively. $L$ and $H$ are scale values corresponding to the "low" and "high" boundaries of the interval (i.e., scale values 0 and 21 in this experiment), and $\mathbf{M}$ is the midpoint between them (here, 10.5). A is a constant whose only purpose is to allow $\mathrm{c}$ to be expressed in the most convenient units. We adopt the convention that the size of $c$ represents the maximum error predicted by Equation 1 and its sign is such that a positive $c$ represents a tendency for responses to fall too close to the midpoint. Another way of describing such a tendency is to say that errors are positive (overestimates) in the numerically lower half of the scale and negative (underestimates) in the numerically higher half.

Any tendency for responses to be too close to the high-numbered end of the interval, say, is assessed by the quadratic coefficient $q$ found by fitting to the data the analogous quadratic functions:

$$
\hat{\mathbf{Y}}=\mathrm{qB}(\mathrm{X}-\mathrm{L})(\mathrm{X}-\mathrm{H}) \text {. }
$$

The size of $q$ is scaled by $B$ to make it represent the largest error predicted by Equation 2, and a positive $\mathrm{q}$ represents a tendency for responses to err towards the low-numbered end of the interval.

Expressions 1 and 2 are plausible components of a model of error, and both reflect the empirical fact that errors tend to be small close to the limits of the interval, but it must be emphasized that their purpose is purely descriptive and does not imply any theoretical commitment to specifically cubic and quadratic functions.

Figure 1 shows a selection of examples to illustrate the relationships linking the values of the $c$ and $q$ coefficients, the shapes of the cubic, quadratic, and combined curves, and the mean errors to which the curves are fitted.

These four sets of data have been selected from our results because they have coefficients which are comparable in absolute magnitude while representing all possible combinations of sign. The proportion of variance in the means accounted for by the combined curve is given by the index $\mathrm{R}^{2}$. The fit of the combined curve was fairly good in these and in most other cases and in only 11 out of the 48 sets of data did it fail to meet a $\mathrm{p}<.001$ level of significance. Six of these 11 cases occurred in condition JV (judgment of a vertical interval), and it may be that the errors on that condition are better described by some other function. Nevertheless, we are satisfied that, in general, our characterization of individual error patterns by a cubic and a quadratic parameter gives an interpretable and valid description. Summaries of our original data have been made available, ${ }^{1}$ but our analysis will concentrate on interpretation of these parameters.

In Figure 2, each subject's quadratic coefficient in the production condition is plotted against his quadratic coefficient in the judgment condition. This is done for both the vertical scale and the horizontal one, giving 24 points on the diagram, two from each subject.

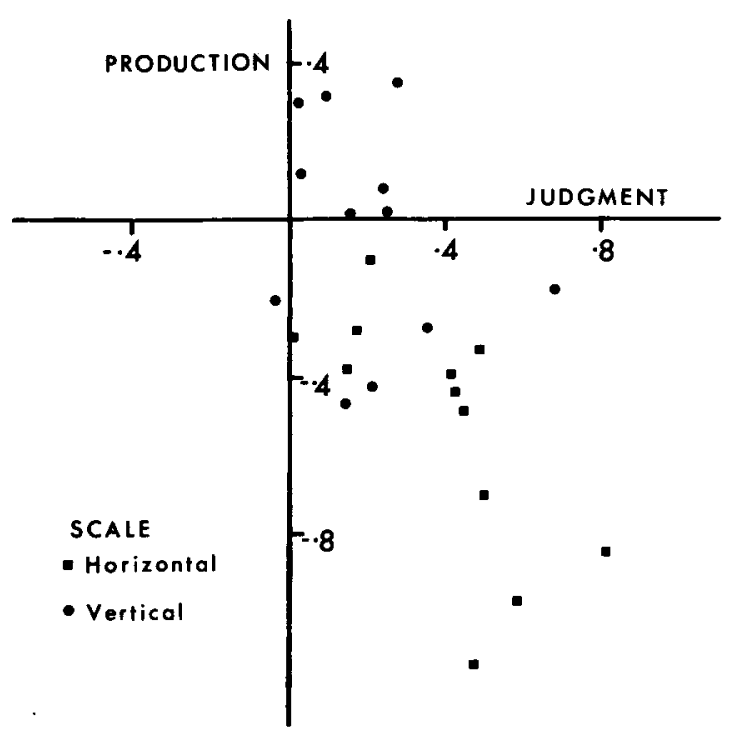

Figure 2. Quadratic coefficients with judgment and production response modes. The magnitude of each coefficient is the maximum of the quadratic curve which is zero at positions 0 and 21 and is a least squares best fit to the errors made by an individual subject on scale positions 1 to 20 . The sign of the coefficient is such that positive coefficients indicate responses which are numerically too small. 
In the judgment conditions, whether the scale was horizontal or vertical, the quadratic components were predominantly positive, showing that errors were mainly numerical underestimates of the true position of the spot. In the production condition, different patterns are found in the two scale types. With the horizontal scale, production resulted in settings which were numerically too high. Not only did all 12 subjects make opposite errors in their judgment and production conditions, but the error sizes were roughly complementary too, resulting in a negative correlation between judgment and production of -.68 , significant at the $5 \%$ level. In other terms, $68 \%$ of the standardized between-subjects variance in the coefficients is common to the two conditions, though it operates in opposite directions.

This result shows that the source of error is perceptual. If it was a bias to prefer responses close to the low-numbered end of the scale, errors would be in the same direction with both response modes. Opposite error directions indicate that errors are made in adjustment because the correct response looks wrong.

With the vertical scale, there is again a negative correlation between judgment and production errors, but the pattern is less clear, perhaps because of a response bias favoring low settings of the spot.

Figure 3 presents the cubic coefficients in the same way. The introduction described how both positive and negative cubic curves have been found with

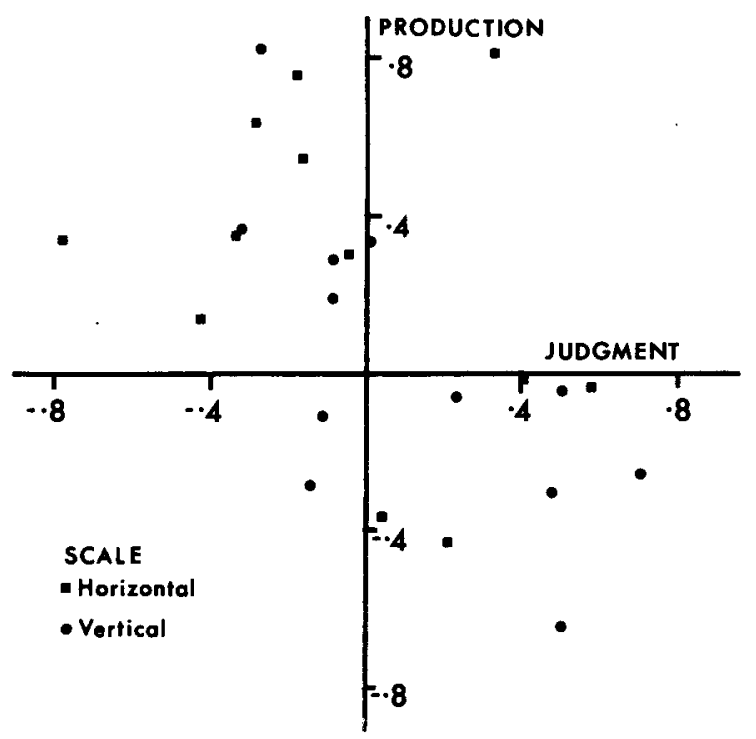

Figure 3. Cubic coefficients with judgment and production response modes. Each coefficient is the maximum of the cubic curve whose value is zero in scale positions $0,10.5$, and 21 and is a least squares best fit to the errors of an individual subject on scale positions 1 to 20. Positive coefficients indicate positive errors in the stimulus range 1 to 10 and negative errors between 11 and 20.

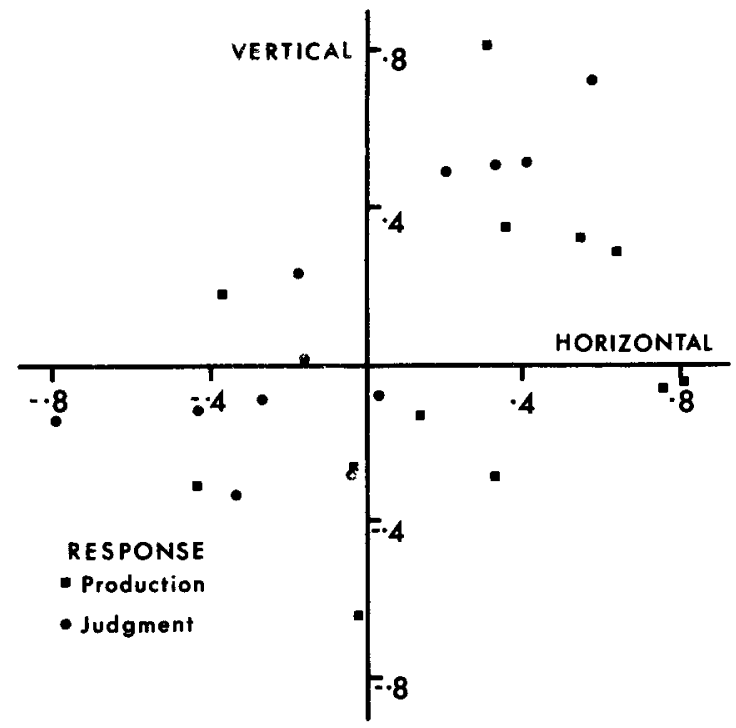

Figure 4. Cubic coefficients with vertical and horizontal linear scales. The coefficients have the same meaning as in Figure 2.

group data. We see here that an individual's judgments may show either type of pattern. The distribution of positive and negative coefficients was similar for the vertical and horizontal scales. Strikingly, however, positive coefficients for judgment go with negative ones for production. There is a negative correlation between the two response modes of -.52 , significant at the $1 \%$ level.

Again, this locates the source of error principally in perception rather than in response biases. Subjects who judge the spot to be nearer to the center than it really is compensate by adjusting it to be further from the center than it should be when attempting to produce a required setting.

There was no evidence that individual differences in error pattern were related to the sex of the subject.

Figure 4 shows the relationship between cubic coefficients on the two types of scale. One of the possible origins of the diverse reported error curves had been thought to be the effect of scale orientation, but it can be seen that the relationship between the two orientations is a positive one, both for judgment and for production $(r=.46$, significant at $5 \%$ ). With few exceptions, subjects maintain the same type of error pattern on both types of scale, thus scale orientation cannot plausibly account for the discrepancies.

\section{DISCUSSION}

It appears that individual subjects have characteristic patterns of perceptual error which persist at least for the minimum of $24 \mathrm{~h}$ covered by this experiment. Although subjects were not tested on the same con- 
dition twice, the negative correlation between judgment and production and the positive one between vertical and horizontal scales would not be predicted if individuals showed haphazard changes in error pattern. These consistent individual differences must cast doubt on any results that represent data pooled over subjects. They suggest, indeed, that differences in reported group patterns may primarily reflect the particular assortment of subjects in the various experiments.

Brooke and MacRae (1977) found similarly consistent individual variation in error pattern when the task was to judge the proportion of horizontals in an array of horizontal and vertical lines. In that case, too, a reversal of pattern was brought about by a change from judgment to production. The similarity between that result and the present one makes it less attractive to seek to explain the results here by invoking a notional "central anchor" in linear scales, as some authors have done. (There could be no central anchor with numerical proportion.) The importance of individual differences makes it dubiously useful to seek general accounts of these error curves in terms of, say, the assimilation or contrast processes proposed by Ward and Lockhead (1970), or indeed using any other explanatory principles which reside essentially in the experimental situation.

A satisfactory account must make reference to the characteristics of individuals, but it cannot be provided yet. We still do not know, for example, whether these patterns of error persist over longer time intervals, nor whether they are consistent across the various types of scale for which cyclic error curves have been reported.

\section{REFERENCES}

Bartlett, N. R., Reed, J. D., \& DuVoisin, G. Estimations of distance on polar coordinate plots as a function of the scale used. Journal of General Psychology, 1949, 41, 47-65.

Brooke, J. B., \& MacRae, A. W. Error patterns in the judgment and production of numerical proportions. Perception \& Psychophysics, 1977, 21, 336-340.

CARR, W. J., \& Garner, W. R. The maximum precision of reading fine scales. Journal of Psychology, 1952, 34, 85-94.

Chapanis, A. Accuracy of interpolation between scale markers as a function of scale interval number. American Psychologist, $1947,2,346$.

Chapanis, A., \& LeyzoreK, M. Accuracy of visual interpolation between scale markers as a function of the number assigned to the scale interval. Journal of Experimental Psychology, 1950, 40, 655-667.

LEVETT, C. M. Errors of interpolation in instrument reading and setting. Journal of Applied Psychology, 1952, 36, 49-52.

LEYZOREK, M. Accuracy of visual interpolation between circular scale markers as a function of the separation between markers. Journal of Experimental Psychology, 1949, 39, 270-279.

Miller, H. K., JR. An exploratory study of linear interpolation. Journal of Applied Psychology, 1950, 34, 367-370.

Nash, H. The judgment of linear proportion. American Journal of Psychology, 1964, 77, 480-484.

WArd, L. M., \& Lockhead, G. R. Sequential effects and memory in category judgments. Journal of Experimental Psychology, 1970, 84, 27-34.

\section{NOTE}

1. A four-page table of mean errors and standard deviations (variable errors) by each subject at each scale value on each condition is available from either author without charge.

(Received for publication September 17, 1976; revision accepted December $17,1979$. 